\title{
Association of Window-side bed Admission with Delirium Suppression in General wards: A Cohort Study of Older Inpatients
}

\section{Daiki Aomura}

Shinshu University

Yosuke Yamada ( $\nabla$ yosukeyama@shinshu-u.ac.jp )

Shinshu University

Makoto Harada

Shinshu University

Koji Hashimoto

Shinshu University

\section{Yuji Kamijo}

Shinshu University

\section{Research Article}

Keywords: Delirium , older inpatients, intensive care, general wards

Posted Date: January 15th, 2021

DOl: https://doi.org/10.21203/rs.3.rs-144531/v1

License: (a) (i) This work is licensed under a Creative Commons Attribution 4.0 International License. Read Full License 


\section{Abstract}

Delirium is a serious problem among older inpatients. Windows in intensive care unit wards may reportedly suppress delirium, with some guidelines recommending the assignment of delirious inpatients to window-side beds. However, whether window-side bed placement suppresses delirium in general wards remains unknown. We therefore performed a retrospective cohort study of 1556 patients who were: 1) admitted to internal medicine departments in Shinshu University Hospital, Japan, between 2009 and 2018 , 2) older than 75 years, 3) admitted to a multi-patient room in a general ward, and 4) admitted unexpectedly. We compared 495 patients assigned to a window-side bed (window group) with 1061 patients assigned to a non-window-side bed (non-window group). The main outcome was delirium with event (e.g. use of anti-delirium medication or physical restraint) within 14 days after admission, which was recorded in 11.1 and 12.1 patients per thousand person-days in the window and non-window group, respectively. Multivariate analysis adjusting for potential confounders including the severity of patient's illness with Cox regression modeling revealed no remarkable association for the window group with outcome development (adjusted hazard ratio 0.90, 95\% confidence interval: 0.61-1.34). Thus, admission to a window-side bed may not prevent delirium in older patients admitted to a general ward.

\section{Introduction}

Characterized by an acute disturbance in mental abilities resulting in confusion, reduced awareness of one's environment, and abnormal behavior ${ }^{1}$, delirium is a serious problem in older inpatients. The condition is usually transient and reversible ${ }^{2}$, but is known to not only physically or mentally harm patients, their family, and medical staff ${ }^{3-5}$, but also increase mortality risk and medical expenses during hospitalization and worsen independence and cognitive function after discharge $\mathrm{e}^{6-11}$. Although delirium mostly develops in inpatients admitted to the intensive care unit (ICU) or after surgery, older inpatients with a medical disease were also reported to exhibit delirium at rates of $6-26 \%^{12}$.

Patients at high risk of delirium may be aided by assignment to a window-side bed on admission. Delirium is closely related to circadian rhythm ${ }^{13}$, and circadian regulation by exposure to sunlight, phototherapy, and melatonin agonists is reportedly effective for suppressing delirium ${ }^{13-15}$. As patients in window-side beds receive considerably more direct exposure to sunlight, their circadian rhythm may be better regulated to prevent delirium. Visibility to the outside through the window is also thought to relax patients and maintain cognition ${ }^{16-18}$. In support of this, several studies have described that the presence of a window in the inpatient room suppressed delirium in the $\mathrm{ICU}^{19}$, and some guidelines recommend to assign patients at high risk of delirium to beds close to a window ${ }^{20}$. However, there is no research studying the impact of window-side placement in patients with a medical disease in general wards.

This study examined the association of admission to a window-side bed with delirium development in older inpatients with a medical disease staying in a multi-patient room in a general hospital ward. 


\section{Methods}

\section{Study design}

This was a retrospective cohort study reviewing the medical charts of patients admitted to Shinshu University Hospital, Japan.

\section{Setting and study population}

The inclusion criteria of this investigation were as follows: 1) admitted to any internal medicine department at Shinshu University Hospital, Japan, between April 2009 and December 2018, 2) admitted to a multi-patient room in a general ward, 3) older than 75 years, and 4) unscheduled or unexpected admission, to identify patients at a higher risk of delirium. The exclusion criteria were as follows: 1) transfer from another hospital, 2) regularly taking anti-delirium medicine before admission, and 3) already delirious on admission. Patients who were transferred from another hospital were excluded due to a lack of data. Anti-delirium medicine was defined as any of haloperidol, quetiapine, perospirone, olanzapine, risperidone, tiapride, mianserin, trazodone, or yokukansan. Delirious at admission was defined as already exhibiting delirium with event, which was the main outcome of this study (described below), within 3 hours after admission.

\section{Baseline characteristics}

The data of eligible patients were collected from hospital medical records and included basic clinical information, sequential organ failure assessment (SOFA) score, performance status (PS), Charlson comorbidity index $(\mathrm{CCl})$, daily medicine use before admission, co-incidence of dementia, and main disease for admission. To calculate SOFA score, we referred to blood test data obtained within 24 hours after admission and predicted the partial pressure of oxygen in arterial blood from peripheral oxygen saturation using the Hill Eq. $2^{1}$. We obtained PS scores as assessed by nurses on patient admission. CCl, the co-incidence of dementia, and the main disease for admission were ascertained using the records of the registered disease name on admission. The main disease for admission was classified as a central nervous system disorder, cardiovascular disease, infection, malignancy, or other.

\section{Exposure of interest}

At Shinshu University Hospital, multi-patient rooms in general wards can accommodate up to 4 or 6 patients. In rooms for 4 patients, the 2 beds closest to the window were defined as window-side beds and the 2 remaining beds were considered non-window-side beds (Fig. 1). In rooms for 6 patients, the 2 beds closest to the window were judged as window-side beds and the remaining 4 beds were defined as nonwindow-side beds. The eligible patients were divided into the group admitted to a window-side bed (window group) or the group admitted to a non-window-side bed (non-window group).

We measured the bedside luminosity in the east ward of the seventh floor using an EM-9300SD device (SATOSHOJI, Japan). Luminosity was considerably higher at window-side beds than at non-window-side 
beds in the daytime, regardless of whether the room faced south or north. Luminosity was undetectable at 9:00 P.M., after lights-out (see Supplementary Fig. S1).

\section{Outcome assessment}

The primary outcome was delirium with event within 14 days after admission. The definition and abstraction method of this outcome are as follows. First, 2 physicians reviewed the medical charts of eligible hospitalized patients and identified delirium development using a reported chart abstraction method $^{22}$. In this method, the physicians searched for key terms indicating acute mental change (e.g., delirium, mental state change, inattention, disorientation, hallucinations, agitation, inappropriate behavior, etc.). If the acute mental change could not be explained by reasons other than delirium (e.g., central nerve system disorder or dementia), the patient was defined as having delirium. Next, the physicians assessed whether the abstracted delirium was accompanied by any of the following events: 1) use of drugs as sedatives for delirium, including anti-delirium medicine, benzodiazepines, and first-generation antihistamines, 2) physical restraint, 3) transfer to another bed, 4) transient stay in the staff room for monitoring, and 5) self-removal of drip line or catheter ${ }^{23,24}$. If the delirium was accompanied by any such event, we classified the case as delirium with event. To enhance the reproducibility and specificity of the main outcome, we selected delirium with event over delirium. The observation period for the primary outcome was limited to 14 days after admission to exclude the influence of a long hospital stay on delirium. Fourteen days was also chosen since Japanese medical staff are basically recommended to discharge patients within 14 days considering that some medical fees are covered by national healthcare for only 14 days of admission.

Both physicians reviewed the medical charts independently and were blinded to whether the patient was in the window or non-window group. If their judgment differed on a primary outcome, mutual consensus was reached by discussion.

We also assessed the secondary outcomes of delirium with event within 30 days after admission, delirium within 14 or 30 days after admission, hospital stay longer than 14 days, transfer to the ICU, and death during hospitalization.

\section{Sample size decision}

Previous literature suggested the primary outcome to occur at a frequency of approximately $10 \%{ }^{12}$. To detect an absolute difference of $5 \%$ in the ratio of the primary outcome between the 2 groups (i.e., $7.5 \%$ in the window group and $12.5 \%$ in the non-window group) with $80 \%$ power at a $5 \%$ significance level, a total of 1280 patients (divided in a 2:3 ratio) were required. Based on this calculation, we set the final recruitment target at 1500 patients.

\section{Statistical analysis}

Descriptive statistics were employed to summarize the demographic factors of the patients stratified by 2 groups. Continuous variables were presented as the median and interquartile range (IQR) and compared 
using the Wilcoxon-Mann-Whitney test. Categorical variables were presented as the number and percentage and assessed by means of the two-tailed $t$-test.

At the assessment of delirium with event or delirium, observation was censored when the following events were recorded for reasons other than delirium: 1) use of anti-delirium medicine, 2) physical restraint, 3) transfer to another bed (apart from window-side bed to window-side bed or non-window-side bed to non-window-side bed), 4) transient stay in the staff room for monitoring, and 5) self-removal of drip line or catheter. Kaplan-Meier curves of cumulative outcome incidence were calculated and compared between the groups using the log-rank test. The hazard ratio of the window group for the main outcome was estimated using multivariable Cox proportional hazard models to adjust for such potential confounders as age, sex, low body weight (i.e., body mass index less than 18.5), SOFA score, regular use of risk drugs for delirium before admission (e.g., benzodiazepines, non-benzodiazepines, anti-histamines, and narcotic analgesics), PS, CCl, admission for central nervous system disorders, and co-incidence of dementia ${ }^{25-27}$. Concerning the assessments of hospital stay for longer than 14 days, transfer to the ICU, and death during hospitalization, the observation period was limited not to 14 days, but to the entire time of hospitalization, and censoring was not taken into account. The adjusted odds ratios of the window group for those outcomes were estimated using logistic regression models.

Multiple imputation was performed to account for missing data values for PS and SOFA scores in 269 patients. We replaced each missing value with a set of substituted plausible values by creating 20 filled-in complete data sets by multiple imputation using a chained equation method ${ }^{28}$. To test the robustness of the results with the multiple imputation method, complete case analysis and median imputation analysis were also performed as sensitivity analyses.

Additional subgroup analyses were conducted using various factors related to the environment of the inpatient and bed, including the type of room, direction of ward, direction of room and window, and season of admission. We assessed the adjusted hazard ratio of the window group for the primary outcome in each subgroup.

All statistical analyses were performed using IBM SPSS statistics version 27.0 (IBM, Armonk, NY). Values of $p<0.05$ were considered statistically significant.

\section{Ethics approval and consent to participate}

This study followed the Strengthening the Reporting of Observational Studies in Epidemiology (STROBE) reporting guidelines. It was performed in accordance with the tenets set forth in the Declaration of Helsinki and approved by the ethics committee of Shinshu University Hospital (authorization number: 4329). Informed written consent was waived in this study by the ethics committee of Shinshu University Hospital due to its retrospective nature using medical records that did not subject the patients to new interventions. The collected data were anonymously stored and used for analysis. As an alternative to written informed consent, an opt-out document was created and posted on the hospital website that 
contained information on the design of the research and publication of the results to provide subjects the opportunity to halt the provision of their medical data.

\section{Results}

\section{Baseline characteristics}

The number of patients fulfilling the inclusion criteria was 1701, among which eligible subjects totaled 1556 after the exclusion of 56 patients (see Supplementary Fig. S2). No patient refused to take part in the study. The characteristics of the patients with and without missing data are described in Supplementary Table S1. The incidence of delirium with event within 14 days after admission was 120 patients $(7.8 \%$; 11.8 per thousand person-days). The breakdown of events was as follows: use of drugs for delirium in 56 cases (46.7\%), physical restraint in 37 cases (30.8\%), transfer to another bed in 12 cases $(10.0 \%)$, transient stay in the staff room in 10 cases $(9.8 \%)$, and self-removal of drip line or catheter in 4 cases (3.3\%).

Regarding the characteristics of the eligible patients, median age was 80.0 years (IQR 77.0-84.0) and the proportion of female was $38.1 \%$. All patients were Japanese. The characteristics of the patients in the window group $(n=495)$ and non-window group $(n=1061)$ are presented in Table 1 . There were no significant differences between the groups for basic characteristics or physical condition, such as SOFA score, PS, and CCI. 
Table 1

Baseline cohort characteristics

\begin{tabular}{|c|c|c|c|}
\hline & $\begin{array}{l}\text { Window group }(n= \\
495)\end{array}$ & $\begin{array}{l}\text { Non-window group }(n= \\
1061)\end{array}$ & $\begin{array}{l}\mathrm{p}- \\
\text { value }\end{array}$ \\
\hline Age, median [IQR], y & $80.0[77.0,84.0]$ & $80.0[77.0,84.0]$ & 0.32 \\
\hline Female, n (\%) & $183(37.0)$ & $410(38.6)$ & 0.54 \\
\hline BMI, median [IQR] & $21.0[18.8,23.6]$ & $21.3[19.0,23.7]$ & 0.34 \\
\hline SOFA score, median [IQR] & $2.0[1.0,3.0]$ & $2.0[1.0,3.0]$ & 0.98 \\
\hline Missing, n (\%) & $16(3.2)$ & $41(3.9)$ & 0.66 \\
\hline \multicolumn{4}{|l|}{$\begin{array}{l}\text { Regular drug use before admission, } \\
\mathrm{n}(\%)\end{array}$} \\
\hline Benzodiazepines & $99(20.2)$ & $208(19.9)$ & 0.89 \\
\hline Non-benzodiazepines & $36(7.4)$ & $97(9.3)$ & 0.24 \\
\hline Anti-histamines & $46(9.4)$ & $88(8.5)$ & 0.56 \\
\hline Narcotic analgesics & $21(4.3)$ & $20(1.9)$ & 0.01 \\
\hline Any of the above drugs & $179(36.2)$ & 349 (32.9) & 0.21 \\
\hline PS, median [IQR] & $0.0[0.0,2.0]$ & $0.0[0.0,1.0]$ & 0.57 \\
\hline Missing, n (\%) & $59(11.9)$ & $162(15.3)$ & 0.09 \\
\hline CCl, median [IQR] & $2.0[1.0,3.0]$ & $2.0[1.0,3.0]$ & 0.78 \\
\hline \multicolumn{4}{|l|}{ Type of main disease, n (\%) } \\
\hline Central nervous system disorder & $38(7.7)$ & $57(5.4)$ & 0.09 \\
\hline Cardiovascular disease & $64(12.9)$ & $130(12.3)$ & 0.74 \\
\hline Infection & $79(16.0)$ & $125(11.8)$ & 0.02 \\
\hline Malignancy & $73(14.7)$ & $166(15.6)$ & 0.71 \\
\hline Other & $241(48.7)$ & $583(54.9)$ & 0.02 \\
\hline Co-incidence of dementia & $13(2.6)$ & $27(2.5)$ & 1.00 \\
\hline
\end{tabular}

\section{Association of window group with primary outcome}

The unadjusted cumulative hazard curves for the primary outcome in the window and non-window groups are shown in Fig. 2. The primary outcome was recorded in 36 cases in the window group (10.7 per thousand person-days) and in 84 cases in the non-window group (11.7 per thousand person-days). Log- 
rank testing did not identify any remarkable difference between the groups $(p=0.78)$. Multivariate analysis with Cox regression models revealed no significant associations for the window group with the primary outcome (adjusted hazard ratio $0.90,95 \%$ confidence interval $0.61-1.34, p=0.62$ ) (Table 2 ). The results of sensitivity analysis on missing data including complete case analysis and median imputation analysis were similar (see Supplementary Table S2).

Table 2

Multivariable analysis on the association of window group and delirium with event

\begin{tabular}{|lcc|}
\hline & Hazard ratio $(95 \% \mathrm{Cl})$ & p-value \\
\hline Crude model & $0.95(0.64$ to 1.40$)$ & 0.78 \\
\hline Adjusted model 1 & $0.96(0.65$ to 1.42$)$ & 0.84 \\
\hline Adjusted model 2 & $0.90(0.61$ to 1.34$)$ & 0.62 \\
\hline $\begin{array}{l}\text { Table shows the hazard ratio of the window group for delirium with event within 14 days after } \\
\text { admission by Cox regression models. In model 1, hazard ratio was adjusted for age and sex. In model } \\
\text { 2, hazard ratio was adjusted for age, sex, low body weight, sequential organ failure assessment score, } \\
\text { regular use of risk drugs for delirium before admission, performance status, Charlson comorbidity } \\
\text { index, admission for central nervous system disorders, and co-incidence of dementia. Abbreviations: } \\
\text { Cl, confidence interval. }\end{array}$ \\
\hline
\end{tabular}

\section{Association of window group with secondary outcomes}

The unadjusted cumulative hazard curves for delirium with event or delirium within 30 days after admission are described in Supplementary Fig. S3. Log-rank testing revealed no significant differences between the groups for delirium with event within 14 days after admission or delirium within 14 or 30 days after admission. Multivariate analysis with a Cox regression model also identified no remarkable associations between the window group and any outcomes (see Supplementary Table S3).

The adjusted odds ratios of the window group for outcomes including hospital stay longer than 14 days, transfer to the ICU, and death during hospitalization were estimated using logistic regression models (see Supplementary Table S4). No significant relationships were observed between the window group and any outcome.

\section{Subgroup analysis on the association of window group with primary outcome}

The results of subgroup analysis are shown in Fig. 3. No significant relationship was present between the window group and the primary outcome of any subgroup. Similarly, no significant interaction effect was detected between the window group and any subgroup.

\section{Discussion}


This is the first study to examine the effects of window-side bed assignment on delirium development in older inpatients admitted to a general ward. As several reports from the ICU found that rooms with a window could suppress delirium ${ }^{19,29,30}$, the admission to window-side beds in general wards was hypothesized to have a similar effect. However, no significant association was found between admission to a window-side bed and delirium development, even after adjusting for possible confounders.

Several reasons may explain why the expected association was absent in this investigation. First, the illnesses of the patients in this study were considerably milder than those of previous reports. According to the ICU research, window placement suppressed delirium by maintaining cognitive function and efficient sleep ${ }^{18}$. However, patients in general wards like those in this study have generally milder illnesses and less frequently experience cognitive dysfunction or sleeping disorders ${ }^{31}$. Thus, it was possible that our cohort did not require such window effects as cognitive function and sleep maintenance, which in turn did not appreciably reduce delirium development. Second, we did not compare rooms with and without windows, but rather compared window-side beds with non-window-side beds. Although earlier studies reported the effect of the presence of a window to suppress delirium by comparing rooms with and without a window ${ }^{19,29,32}$, those results could have been caused not by the window itself, but by window-associated accompanying factors, such as the newness and clarity of the room. The present study compared window-side beds with non-window-side beds in identical layout rooms of a single center, which was considered to assess the direct impact of windows on delirium through sunlight exposure and visibility of the outside world. Indeed, almost half of the ICU research reported that windows did not decrease the development of delirium ${ }^{33}$, implying the possibility that the simple presence of a window did not associate with delirium prevention. Furthermore, we did not identify any significant associations for window-side beds with delirium in subgroup analyses factoring the environments of patients or rooms. These results support the result that windows do not directly impact delirium. Third, it was conceivable that our study's statistical power was insufficient to detect differences between the test groups. The risk difference in the ratio of the primary outcome was $0.6 \%(7.3 \%$ in the window group and $7.9 \%$ in the non-window group) in this study, which was considerably less than the predicted $5 \%$. Thus, the effect of a window-side bed on delirium suppression was much smaller than our estimation, and hence our sample size might not have been sufficient to detect an effect. Additional research with a larger sample size is needed for further assessment.

This study had several limitations that must be considered when interpreting our results. First, because we retrospectively abstracted delirium development referring to medical charts, the diagnostic accuracy of delirium was presumed as not completely accurate. In retrospective studies on delirium, the chart abstraction method by Inouye et al. is generally used ${ }^{22}$ with a sensitivity and specificity of $73 \%$ and $84 \%$, respectively. In the present research, however, diagnosis by this method may have had diminished accuracy since the reviewers were not delirium specialists. To enhance our diagnostic accuracy, however, we additionally assessed whether the abstracted delirium was accompanied by intervention, and so delirium with event was abstracted as a more reproducible and specific outcome. Although this assessment method likely could not sufficiently abstract mild or hypoactive delirium, we considered that 
delirium with event would be more suitable as the main outcome presuming that non-specialists of delirium basically observe, manage, and treat severe or hyperactive delirium cases more frequently than

mild or hypoactive cases in general wards ${ }^{34}$. We also evaluated the association of the window group with delirium defined by the chart abstraction method, and again no significant relationship was found, implying high reproducibility of this research. Second, there was the possibility that unmeasured confounding factors influenced the results. We could not abstract some potential confounders, including family structure of the patient, catheterization during hospitalization, and patient bed location request. Moreover, the number of patients in the window group was much smaller than in the non-window group, even after considering the difference in numbers of window-side and non-window-side beds. Although we assume this imbalance is due to the differences in hospitalization period and bed turnover rate between the groups, it cannot be denied that patient assignment was influenced by unmeasured confounders. Lastly, the external validity of this research is limited due to its design as a single-center study at a university hospital. It is unclear whether our results can be applied for other patients, especially those at long-term hospitals or nursing homes, because their characteristics and hospitalization environments differ considerably. Additional multi-center studies are required to confirm the external validity of our findings.

In summary, this study revealed no significant association between admission to a window-side bed and delirium development in older patients with a medical disease in a general ward. Larger multi-center studies are warranted to refine and validate our results.

\section{Declarations}

\section{Data availability}

All data analyzed during the current study are available from the first author on reasonable request.

\section{Author contributions}

D.A. has full access to all of the data in this research and takes responsibility for the integrity of the data and the accuracy of the data analysis. D.A. designed the study and drafted the article with support from Y.Y.. D.A. and Y.Y. checked the medical charts and assessed the incidence of delirium. M.H. and K.H. revised the article critically for important intellectual content. Y.Y. and Y.K. revised the article critically for important intellectual content and gave final approval of the submitted version.

\section{Conflicts of interest:}

The authors declare no conflicts of interest. 


\section{Competing interests:}

This article was supported by a grant from the Shinshu Public Utility Foundation for Promotion of Medical Sciences.

\section{References}

1. Herling, S. F. et al. Interventions for preventing intensive care unit delirium in adults. Cochrane Database Syst Rev 11, Cd009783, doi:10.1002/14651858.CD009783.pub2 (2018).

2. Wang, H. R., Woo, Y. S. \& Bahk, W. M. Atypical antipsychotics in the treatment of delirium. Psychiatry Clin Neurosci. 67, 323-331 https://doi.org/10.1111/pcn.12066 (2013).

3. Partridge, J. S., Martin, F. C., Harari, D. \& Dhesi, J. K. The delirium experience: what is the effect on patients, relatives and staff and what can be done to modify this?. Int J Geriatr Psychiatry. 28, 804812 https://doi.org/10.1002/gps.3900 (2013).

4. Martins, S. et al. What effect does delirium have on family and nurses of older adult patients?. Aging Ment Health. 22, 903-911 https://doi.org/10.1080/13607863.2017.1393794 (2018).

5. Schmitt, E. M. et al. Perspectives on the Delirium Experience and Its Burden: Common Themes Among Older Patients, Their Family Caregivers, and Nurses. Gerontologist. 59, 327-337 https://doi.org/10.1093/geront/gnx153 (2019).

6. Ely, E. W. et al. Delirium as a predictor of mortality in mechanically ventilated patients in the intensive care unit. Jama. 291, 1753-1762 https://doi.org/10.1001/jama.291.14.1753 (2004).

7. Pisani, M. A. et al. Days of delirium are associated with 1-year mortality in an older intensive care unit population. Am J Respir Crit Care Med. 180, 1092-1097 https://doi.org/10.1164/rccm.200904$05370 C$ (2009).

8. Inouye, S. K. \& Charpentier, P. A. Precipitating factors for delirium in hospitalized elderly persons. Predictive model and interrelationship with baseline vulnerability. Jama. 275, 852-857 (1996).

9. Rudolph, J. L. et al. Delirium: an independent predictor of functional decline after cardiac surgery. $J$ Am Geriatr Soc. 58, 643-649 https://doi.org/10.1111/j.1532-5415.2010.02762.x (2010).

10. Girard, T. D. et al. Delirium as a predictor of long-term cognitive impairment in survivors of critical illness. Crit Care Med. 38, 1513-1520 https://doi.org/10.1097/CCM.0b013e3181e47be1 (2010).

11. Girard, T. D., Pandharipande, P. P. \& Ely, E. W. Delirium in the intensive care unit. Crit Care. 12 Suppl (3), S3 https://doi.org/10.1186/cc6149 (2008).

12. Lindesay, J., Rockwood, K. \& Macdonald, A. in Delirium in Old Age (ed James Lindesay) 3135(Oxford University Press, 2002).

13. Oldham, M. A., Lee, H. B. \& Desan, P. H. Circadian Rhythm Disruption in the Critically III: An Opportunity for Improving Outcomes. Crit Care Med. 44, 207-217 https://doi.org/10.1097/ccm.0000000000001282 (2016). 
14. Ono, H., Taguchi, T., Kido, Y., Fujino, Y. \& Doki, Y. The usefulness of bright light therapy for patients after oesophagectomy. Intensive and Critical Care Nursing. 27, 158-166 https://doi.org/10.1016/j.iccn.2011.03.003 (2011).

15. Potharajaroen, S. et al. Bright light and oxygen therapies decrease delirium risk in critically ill surgical patients by targeting sleep and acid-base disturbances. Psychiatry Res. 261, 21-27 https://doi.org/10.1016/j.psychres.2017.12.046 (2018).

16. Ulrich, R. S. Visual landscapes and psychological well-being. Landscape Research. 4, 17-23 https://doi.org/10.1080/01426397908705892 (1979).

17. Ulrich, R. S. View through a window may influence recovery from surgery. Science. 224, 420-421 https://doi.org/10.1126/science.6143402 (1984).

18. Criner, G. J. \& Barnette, R. E. \& D'Alonzo, G. E. Critical care study guide: text and review. (2010).

19. Zaal, I. J. et al. Intensive care unit environment may affect the course of delirium. Intensive Care Med. 39, 481-488 https://doi.org/10.1007/s00134-012-2726-6 (2013).

20. Aisha Lofters, M., Frayha, N. \& Primary Care, R. A. P. October 2020 Written Summary. Imaging 6, 217229 (2015).

21. Hill, A. \& PROCEEDINGS OF THE PHYSIOLOGICAL SOCIETY: January 22, 1910. The Journal of Physiology 40, i-vii, doi:10.1113/jphysiol.1910.sp001386 (1910).

22. Inouye, S. K. et al. A chart-based method for identification of delirium: validation compared with interviewer ratings using the confusion assessment method. J Am Geriatr Soc. 53, 312-318 https://doi.org/10.1111/j.1532-5415.2005.53120.x (2005).

23. Johansson, Y. A., Bergh, I., Ericsson, I. \& Sarenmalm, E. K. Delirium in older hospitalized patientssigns and actions: a retrospective patient record review. BMC Geriatrics. 18, 43 https://doi.org/10.1186/s12877-018-0731-5 (2018).

24. Dubois, M. J., Bergeron, N., Dumont, M., Dial, S. \& Skrobik, Y. Delirium in an intensive care unit: a study of risk factors. Intensive Care Med. 27, 1297-1304 https://doi.org/10.1007/s001340101017 (2001).

25. Yang, J. et al. Risk Factors of Delirium in Sequential Sedation Patients in Intensive Care Units. BioMed Research International 2017, 3539872, doi:10.1155/2017/3539872 (2017).

26. Boltz, M. Evidence-based geriatric nursing protocols for best practice(Springer Pub. Co., 2012).

27. Ahmed, S., Leurent, B. \& Sampson, E. L. Risk factors for incident delirium among older people in acute hospital medical units: a systematic review and meta-analysis. Age Ageing. 43, 326-333 https://doi.org/10.1093/ageing/afu022 (2014).

28. Aloisio, K. M., Swanson, S. A., Micali, N., Field, A. \& Horton, N. J. Analysis of partially observed clustered data using generalized estimating equations and multiple imputation. Stata J. 14, 863-883 (2014).

29. Wilson, L. M., Intensive Care \& Delirium The Effect of Outside Deprivation in a Windowless Unit. Archives of Internal Medicine. 130, 225-226 
https://doi.org/10.1001/archinte.1972.03650020055010 (1972).

30. Keep, P., James, J. \& Inman, M. Windows in the intensive therapy unit. Anaesthesia. 35, 257-262 https://doi.org/10.1111/j.1365-2044.1980.tb05093.x (1980).

31. Altman, M. T. et al. Association of intensive care unit delirium with sleep disturbance and functional disability after critical illness: an observational cohort study. Annals of Intensive Care. 8, 63 https://doi.org/10.1186/s13613-018-0408-4 (2018).

32. Van Rompaey, B. et al. Risk factors for delirium in intensive care patients: a prospective cohort study. Crit Care. 13, R77 https://doi.org/10.1186/cc7892 (2009).

33. Hashemighouchani, H. et al. The Impact of Environmental Risk Factors on Delirium and Benefits of Noise and Light Modifications: A Scoping Review. medRxiv 9, 1183, doi:10.1101/2020.05.20.20108373 (2020).

34. Inouye, S. K., Foreman, M. D., Mion, L. C., Katz, K. H. \& Cooney, L. M. Jr. Nurses' recognition of delirium and its symptoms: comparison of nurse and researcher ratings. Arch Intern Med. 161, 2467-2473 https://doi.org/10.1001/archinte.161.20.2467 (2001).

\section{Figures}




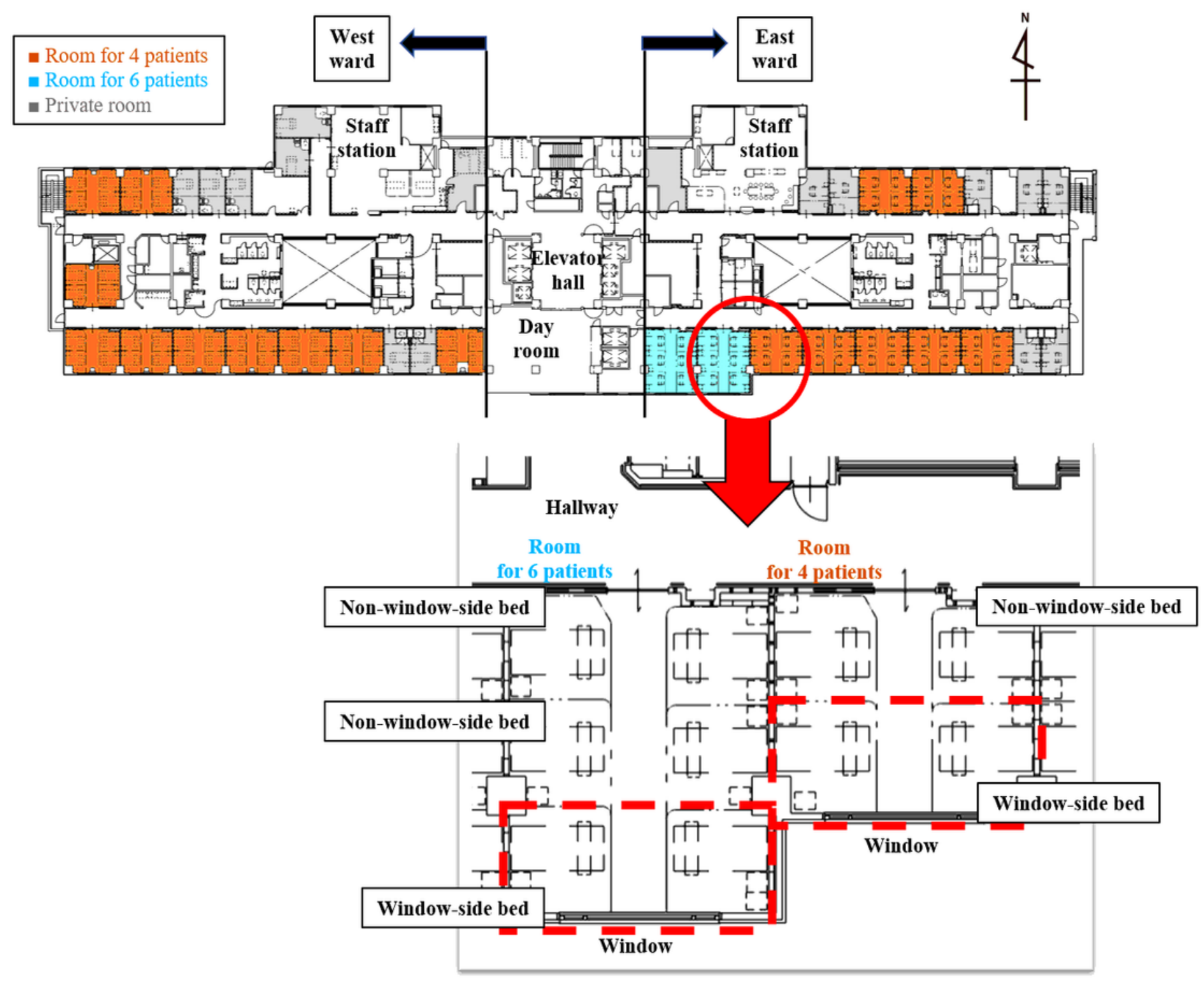

Figure 1

Map of a general hospital ward The map shows the layout of a general ward at Shinshu University Hospital. There are 2 types of multi-patient rooms: rooms for 4 patients and rooms for 6 patients. In both room types, the 2 beds nearest to the window are designated window-side beds (red dotted lines), while the others are designated non-window-side beds. 


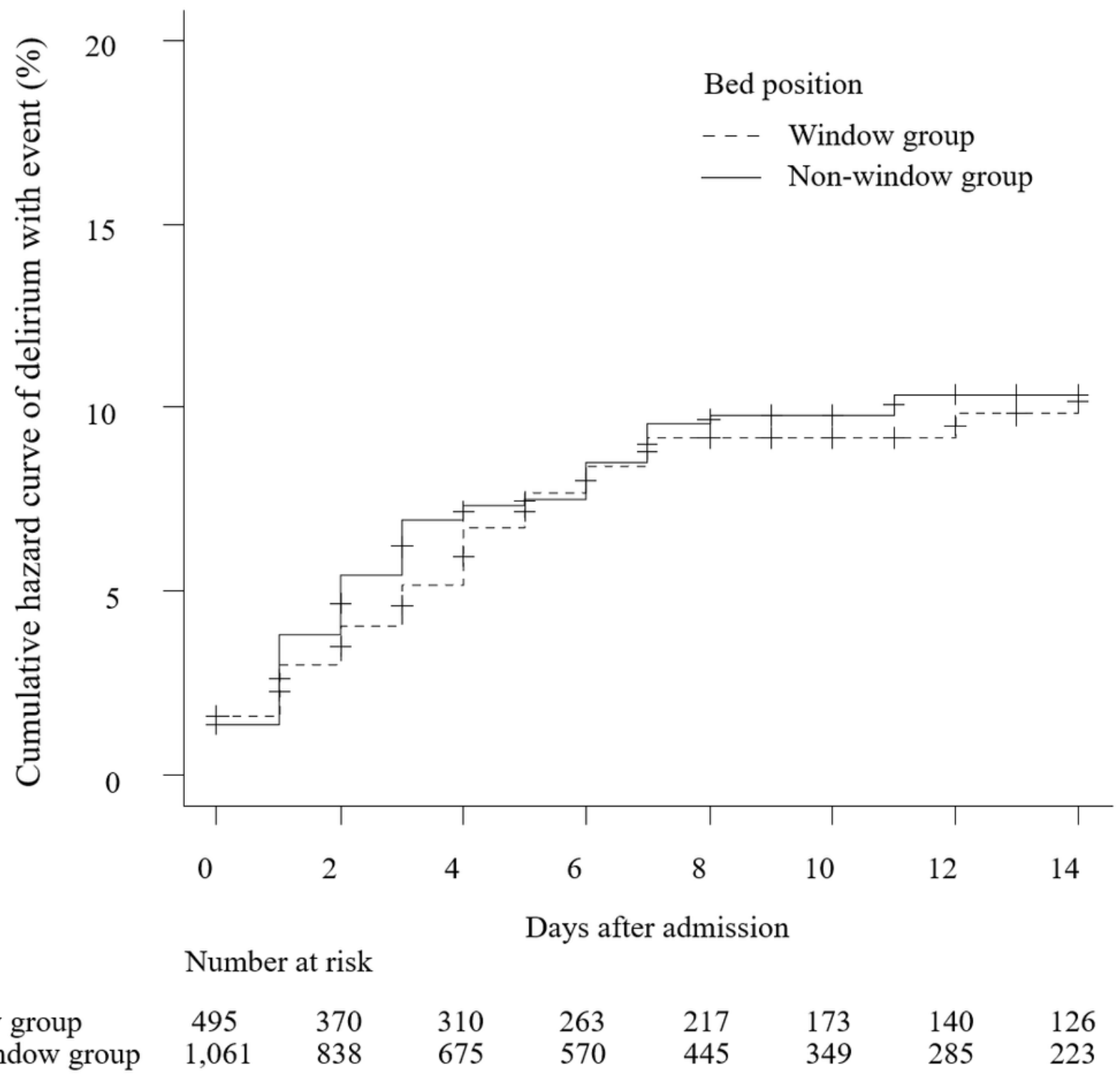

\section{Figure 2}

Unadjusted cumulative curves for the primary outcome The figure shows the cumulative curves of delirium with event within 14 days after admission. The dotted line indicates the window group and the solid line indicates the non-window group. 


\begin{tabular}{|c|c|c|c|c|}
\hline & Patients, n (\%) & Adjusted HR $(95 \% \mathrm{CI})^{a}$ & p-value & p-value for interaction \\
\hline Overall & 1,556 & $0.90(0.61-1.34)$ & 0.62 & \\
\hline \multicolumn{5}{|l|}{ Room type } \\
\hline Room for 4 patients & $1,295(83.2)$ & $0.92(0.60-1.40)$ & 0.71 & 0.91 \\
\hline Room for 6 patients & $261(16.8)$ & $0.80(0.22-2.89)$ & 0.74 & \\
\hline \multicolumn{5}{|l|}{ Direction of ward } \\
\hline East ward & $926(59.5)$ & $0.86(0.50-1.47)$ & 0.59 & 0.69 \\
\hline West ward & $630(40.5)$ & $0.86(0.50-1.47)$ & 0.82 & \\
\hline \multicolumn{5}{|c|}{ Direction of room and window } \\
\hline Facing south & $640(41.1)$ & $0.84(0.45-1.56)$ & 0.59 & 0.76 \\
\hline Facing north or west & $916(58.9)$ & $0.90(0.53-1.52)$ & 0.70 & \\
\hline \multicolumn{5}{|l|}{ Season of admission } \\
\hline Spring or summer & $793(51.0)$ & $0.61(0.34-1.10)$ & 0.10 & 0.08 \\
\hline Autumn or winter & $763(49.0)$ & $1.26(0.72-2.20)$ & 0.41 & \\
\hline
\end{tabular}

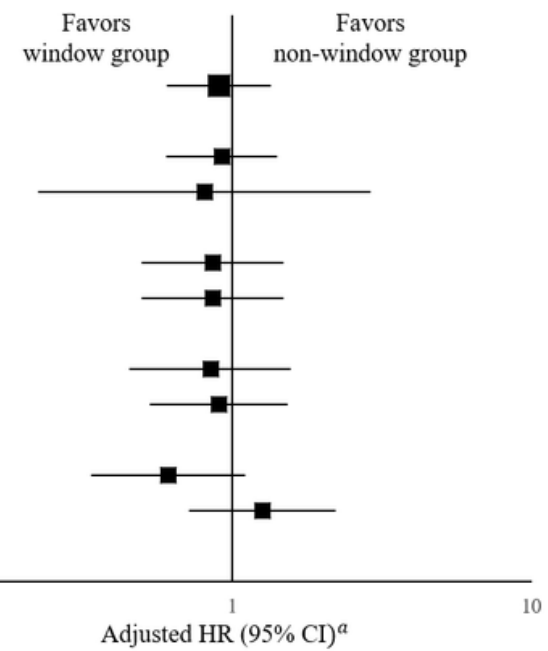

\section{Figure 3}

Subgroup analysis on the association of window group with primary outcome The Table and forest plot show the adjusted hazard ratio of the window group for delirium with event within 14 days after admission for each subgroup. In the forest plot, black squares show the adjusted HR and horizontal lines show $95 \%$ Cls. a HR was modified using the Cox regression model adjusted for age, sex, low body weight, sequential organ failure assessment score, regular use of risk drugs for delirium before admission, performance status, Charlson comorbidity index, admission for central nervous system disorders, and coincidence of dementia. Abbreviations: $\mathrm{HR}$, hazard ratio; $\mathrm{Cl}$, confidence interval.

\section{Supplementary Files}

This is a list of supplementary files associated with this preprint. Click to download.

- Onlineonlymaterialsjan132021.docx 